\title{
Communication \\ Selective Disinfection Based on Directional Ultraviolet Irradiation and Artificial Intelligence
}

\author{
Ben Zierdt ${ }^{1}$, Taichu Shi ${ }^{1}$, Thomas DeGroat ${ }^{1}$, Sam Furman ${ }^{1}$ (D), Nicholas Papas ${ }^{1}$, Zachary Smoot ${ }^{1}$, Hong Zhang ${ }^{2}$ \\ and Ben $\mathrm{Wu}^{1, *}$ \\ 1 Department of Electrical and Computer Engineering, Rowan University, 201 Mullica Hill Rd., \\ Glassboro, NJ 08028, USA; zierdtb9@students.rowan.edu (B.Z.); shitai32@rowan.edu (T.S.); \\ degroatt2@students.rowan.edu (T.D.); furmans5@students.rowan.edu (S.F.); \\ papasn7@students.rowan.edu (N.P.); smootz6@students.rowan.edu (Z.S.) \\ 2 Department of Mechanical Engineering, Rowan University, 201 Mullica Hill Rd., Glassboro, NJ 08028, USA; \\ zhang@rowan.edu \\ * Correspondence: wub@rowan.edu
}

check for

updates

Citation: Zierdt, B.; Shi, T.; DeGroat, T.; Furman, S.; Papas, N.; Smoot, Z.; Zhang, H.; Wu, B. Selective

Disinfection Based on Directional Ultraviolet Irradiation and Artificial Intelligence. Electronics 2021, 10, 2557. https://doi.org/10.3390/electronics 10202557

Academic Editor: Antonio Di Bartolomeo

Received: 25 August 2021

Accepted: 14 October 2021

Published: 19 October 2021

Publisher's Note: MDPI stays neutral with regard to jurisdictional claims in published maps and institutional affiliations.

Copyright: (c) 2021 by the authors. Licensee MDPI, Basel, Switzerland. This article is an open access article distributed under the terms and conditions of the Creative Commons Attribution (CC BY) license (https:// creativecommons.org/licenses/by/ $4.0 /)$.

\begin{abstract}
Ultraviolet disinfection has been proven to be effective for surface sanitation. Traditional ultraviolet disinfection systems generate omnidirectional radiation, which introduces safety concerns regarding human exposure. Large scale disinfection must be performed without humans present, which limits the time efficiency of disinfection. We propose and experimentally demonstrate a targeted ultraviolet disinfection system using a combination of robotics, lasers, and deep learning. The system uses a laser-galvo and a camera mounted on a two-axis gimbal running a custom deep learning algorithm. This allows ultraviolet radiation to be applied to any surface in the room where it is mounted, and the algorithm ensures that the laser targets the desired surfaces avoids others such as humans. Both the laser-galvo and the deep learning algorithm were tested for targeted disinfection.
\end{abstract}

Keywords: ultraviolet disinfection; laser-galvo system; COVID-19; automatic control

\section{Introduction}

Due to the COVID-19 (SARS-CoV-2) pandemic, the importance of maintaining clean surfaces through frequent disinfection and sterilization have been especially stressed, both at home and in commercial spaces. Ultraviolet (UV) irradiation has proven to be an effective disinfection tool that deactivates the RNA structure of the virus. Recent studies after the outbreak of SARS-CoV-2 have shown that the applications of UV radiation are viable for disinfecting surfaces in heavily utilized environments [1]. Similarly, a statistical analysis of different studies that utilized a UV application to inactivate SARS-CoV-2 found that, in a highly controlled test, the inactivation/eradication of the virus is dependent on the UV dose and concentration of the virus [2,3]. Data has led to the conclusion that UV irradiation is an effective disinfection method [4], allowing the team to continue developing a tool to combat SARS-CoV-2, in addition to other viruses, using forms of UV irradiation.

\subsection{Virus Disinfection}

As shown in various applications, UV irradiation has been shown to be effective at inactivating viruses on various surfaces, including water, plastic, metal, and air. Specifically, the UV-C band (200-280 nm) has shown to be the most effective at inactivating viruses [2]. Viruses are inactivated by shining UV radiation with a specific wavelength on the virus. Although this does not kill it, it essentially renders it useless because it is unable to replicate and thus survive. The method by which this is calculated is via the dosage. Dosage is equivalent to irradiance $\left(\mathrm{mW} / \mathrm{cm}^{2}\right)$ times exposure time (s) [3]. Hence, most journals refer to the dosage needed to inactivate viruses rather than just the time, because both components are required to properly inactivate a virus. It is in this manner of disinfection 
that our system prevails because it greatly reduces the required time. This is because the power transmitted to a large area is much greater in the laser beam medium compared to an UV LED.

Using the focused power of a laser beam, it only takes a few seconds or less to finish the disinfection on the contaminated surface. This process generates zero irradiation to surrounding humans. It was shown that a UV laser can be up to $200 \times$ more powerful than an UV LED while still emitting the same desired wavelength $[5,6]$. This means that a more powerful effect of disinfection can be achieved because dose correlates to disinfection. Additional benefits of a targeted disinfection system with lasers compared to LEDs are its single wavelength, application optimization, and consistent output $[7,8]$.

\subsection{Laser vs. Diode//Commerical UV Disinfection Use}

Traditionally, UV LEDs are used to disinfect in the form of UV lamps. This is because they can be mobile at smaller scales, and disinfect everything in sight. In particular applications, such as water supplies, this method functions relatively well. However, because the existing UV disinfection methods deploy UV lamps with omnidirectional irradiations, they have two main limitations: (1) With UV power irradiated in all directions, the average power per unit area can be lacking, and it takes several hours to accumulate enough UV power to perform the needed dose [9]. With the explosion of SARS-CoV-2, there is an urgent need for rapid disinfection, meaning this approach is flawed. (2) The omnidirectional irradiations indiscriminately attack both the virus and humans alike [10]. An indoor area must be vacant before disinfection, which further limits the efficiency of UV disinfection with this application. Additionally, because this approach takes several hours to accomplish, quick disinfection is not feasible with lamps. Hence, our team was motivated to make a novel UV disinfection system based on a UV laser rather than an LED.

This method has significant benefits. Some of the benefits of using a UV laser include more focused power, less overall power needed, greater efficiency, and being less harmful. UV lasers also last longer than the UV lamps, which can have as few as 10,000 $\mathrm{h}$ of operation before needing to be replaced [9]. However, this method of disinfection is particularly useful due to the focused power distribution of the laser beam. This allows for disinfection in the presence of humans given the proper theoretical laser setup and safety considerations, both of which are outlined below.

\subsection{Theoretical Laser Specifications}

In order to properly implement the proposed UV laser solution, certain specifications must be met. The doses needed to deactivate viruses vary, and can range from 10.25 to $23.71 \mathrm{~mJ} / \mathrm{cm}^{2}$ [1]. However, these tests were conducted with UV lamps at the $254 \mathrm{~nm}$ wavelength with a surface power density of $0.466 \mathrm{~W} / \mathrm{cm}^{2}$. Although the principle is similar to that when using a laser, several other characteristics must also be considered.

This disinfection process was also undertaken experimentally with a laser in a different source which had monochromatic, coherent, and linearly polarized laser properties, and was then collimated into a beam. This laser had an output power of $1.1 \mathrm{~mW}$, energy density of $10 \mathrm{~mJ} / \mathrm{cm}^{2}$, and the targeted wavelength range for disinfection is $205-230 \mathrm{~nm}$ [9].

Therefore, we proposed a laser that is capable of performing the required dosages in a smaller amount of time. A laser with a frequency in the UV-C band is optimal for inactivating viruses. The laser must also be able to meet the irradiance requirement that allows for rapid disinfection. With an energy density of $10 \mathrm{~mJ} / \mathrm{cm}^{2}$ and power of at least $1 \mathrm{~mW}$, it would be possible to disinfect viruses relatively quickly (in the order of $0.025 \mathrm{~s}$ ). However, because the spectrum of the UV-C band required $(200-280 \mathrm{~nm}$ ) is beyond human visibility, for our experimental setup, we instead used a violet light for testing. Because our system is meant to test the mobility of the laser and the ability to target specific classes, rather than the actual inactivation of viruses, our system test did not require the theoretical UV-C spectrum or a laser capable of reaching the UV-C wavelength. The experimental 
system used is described in the following sections and comprised hardware and software subsystems that were interdependent of each other.

\subsection{Hardware Subsystem}

Our hardware system is comprised of the physical laser source, a power supply, a beam expander, a two-dimensional galvo mirror, and its control circuit. As mentioned previously, the laser in our setup is simply a violet laser to ensure visibility and not a UV-C laser required for actual disinfection. The galvo mirror is used to control the direction of the irradiation. The disinfection system is able to scan the surface at a speed of 100 cycles/second. The disinfection system controls the direction of a laser with the twodimensional galvo-mirrors. The galvo system is able to scan both " $x$ " and " $y$ " directions. It is also possible to mount the disinfection system on various moving platforms, such as a drone, to improve the range of disinfection coverage. Additional considerations for the actuating mechanism were accounted for, such as versatility and compatibility [11]. Other hardware considerations, such as a universal mounting bracket or autonomous navigating body, were considered based on the application of UV disinfection.

\subsection{Software Subsystem}

Artificial intelligence (AI) is used to analyze the image from a camera sensor, identify the surface that requires disinfection, and avoid human exposure to UV irradiation. A selective disinfection and sterilization system increases the efficiency of disinfecting a given area and, with proper measures, also increases the overall safety $[12,13]$. This in turn enables the system to be "smart", where it can function autonomously and perform disinfection to the contaminated surfaces while avoiding exposure to humans. An autonomous system capable of discerning whether an object needs to be disinfected, or prevented from being exposed to a laser, allows the system to be used at any time of day and in more scenarios compared to the UV LED application mentioned above.

In order to identify the surface that needs to be disinfected, the team trained a neural network using TensorFlow to recognize different classes [14,15]. This was accomplished using images that resulted in a relatively reliable success rate, which allows it to detect common surfaces (that would need to be disinfected) and humans (to avoid unnecessary laser exposure). Additionally, software to control the mechanical systems was developed to allow the disinfection system to be able to direct and focus the laser on designated targets (that the AI algorithm detected). In total, the AI algorithm and the controlling software function simultaneously to identify the contaminated surface and perform selective disinfection.

\section{System Implementation}

\subsection{System Overview}

The whole procedure is divided into three main steps (shown in Figure 1c). The first is to collect the information of the area through the camera. The second is to perform image recognition through a deep learning network to determine which parts of the scanned area need to be disinfected. If a human is detected in this step, the whole process is stopped immediately. Finally, according to the result of the previous step, the galvanometer system is driven to scan the specific area and complete the targeted disinfection. Figure 1a shows the galvanometer system setup mounted on a movable cart in our experiment. This combination allows for the most degrees of freedom to allow a large field of view for disinfection, even from a stationary location. When the process starts, the UV laser is expanded by the beam expander to cover the entire galvo mirror. The speed and trajectory of laser beam movement can also be adjusted by the galvanometer. The galvanometer can be further controlled by a deep learning algorithm through a computer. Figure $1 \mathrm{~b}$ shows the result of the laser beam on a certain target. As shown in Figure 1b, by controlling the angle of the galvanometer, the laser can be very accurately focused on a certain target. The intensity at this focal point is much greater than that of a general UV LED/lamp. As the 
galvanometer system starts to vibrate, the focus can quickly scan according to a preset trajectory to achieve the purpose of rapid disinfection.

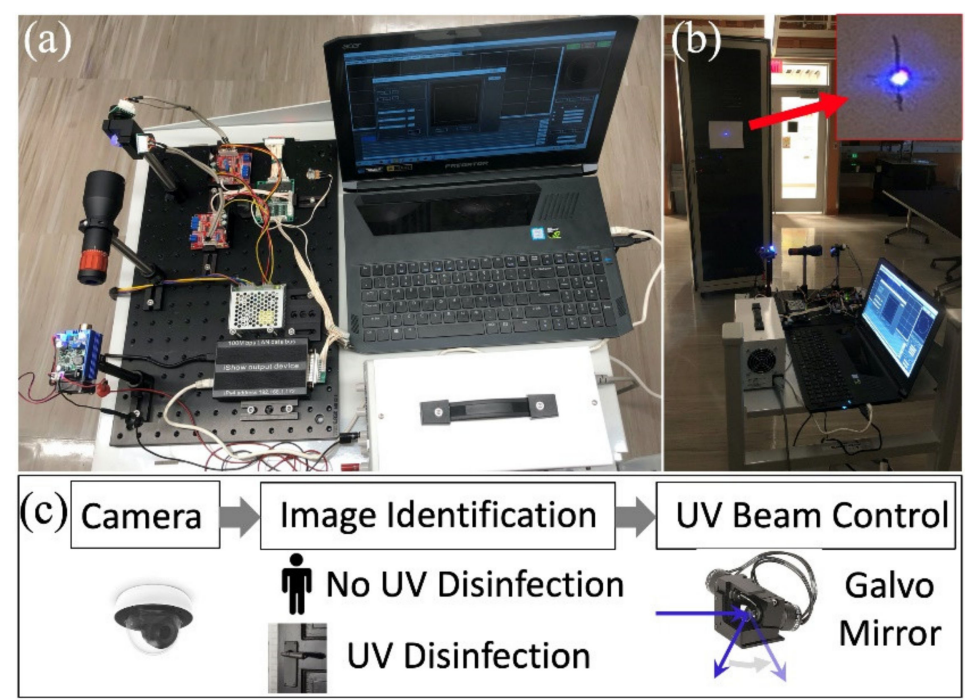

Figure 1. (a) Prototype on a moving cart; (b) system test with UV laser on; (c) system flowchart.

\subsection{Deep Learning Algorithm}

The purpose of the deep learning algorithm in this project is to determine whether a specific target needs to be disinfected. This can be achieved through image recognition technology. After training the deep learning model, the system can identify several classes of objects for the primary goals of either sanitizing or avoiding sanitization depending on the object. The image recognition system was developed using several classes of common objects that would often be present in daily life. More classes for detecting and disinfecting specific targets can also be added to the network model for training. The classes used in this project are listed below.

Table 1 shows the classes that the algorithm was trained to detect and disinfect. However, class 8 was added, i.e., training to detect humans, to ensure that a person is not disinfected at all. This is one of the more important classes because it acts as an emergency stop button. If a person appears in the detected scene, then all other class categories will be overridden and the whole system will turn off immediately, rather than attempting to disinfect another class that is in front of the person.

Table 1. List of image classes used in this project.

\begin{tabular}{cc}
\hline Number of Classes & Label Name \\
\hline 1 & Light switch \\
2 & Door handle \\
3 & Chair \\
4 & Table/Desk \\
5 & Counter-top \\
6 & Computer mouse \\
7 & Computer keyboard \\
8 & Person \\
\hline
\end{tabular}

For training processes, we used the SSD ResNet50 V1 FPN $640 \times 640$ network model. This is a residual neural network with 50 layers, including 48 associated convolutional layers, one MaxPool layer, and one average pool layer [16-18]. Compared with the traditional convolutional neural network, it solves the problem of gradient disappearance caused by increasing depth in the deep neural network, so it can obtain deeper image features, thereby making the prediction results more accurate. The inputs of this network model are 
images scaled to $640 \times 640$ resolution from a single shot detector (SSD). The convolutional layer can be used to extract the features of the objects in the picture. The pooling layer can be used to select these features and retain the most important features of the object for identifying its classification. Image recognition technology based on residual network is relatively mature, and its prediction accuracy is very high. Because it contains many convolution layers, and the weights in the convolutional layers are also related, the input images can be divided into smaller grids for feature extraction. The features extracted in this manner not only contain more information, but also have higher accuracy, which can provide very precise control guidance for our galvo mirror system. The basic training parameters of this neural network included a batch size of 8, 25,000 steps, a learning rate of 0.04 and a maximum number of boxes of 100. Furthermore, each class contained 1000 samples to give the system a suitable baseline for identification. Our training model had initial weights loaded from a model trained on the COCO 2017 dataset [19] and images resized to $640 \times 640$. In our dataset, $90 \%$ of the images from each class were used for the model training, whereas the remaining $10 \%$ was used for self-evaluation. In this manner, the network model can be prevented from overfitting, but it is not universal and robust to general scenes $[20,21]$. Once the training is completed, the entire system can complete fast offline predictions. If more new recognition targets are needed, only the model needs to be retrained. This neural network yielded acceptable results, as discussed further in the Results and Analysis section.

\subsection{Saftey Considerations}

The primary concerns regarding the safety of this setup include the risk to humans entering the range of the disinfection area. Given the harmful nature of UV irradiation to human health, it is paramount that there be no contact with any surrounding humans. This is considered in our deep learning algorithm. Because our system scans the area and identifies the different classes, if a human is detected it will not disinfect. An example is shown later in the Results and Analysis section, in which a human is sitting on a stool, and the proposed approach does not disinfect the stool. It is also able to detect when sufficiently far away, and disinfect another class item, such as a doorknob, in the presence of a human. This allows frequent and safe disinfection of common items, while avoiding the problem of unintended people being in the path of the laser beam.

Another consideration is that of reflections. This is an important aspect because, in order to ensure the safety of those surrounding the setup, proper care must be taken to detect reflections. Deep learning is capable of addressing this issue. Although it is more complicated than simply adding a class, it is something that would be attainable with future improvements to the deep learning algorithm, in addition to considering alternative means of detection, such as detecting some of the radiation that may reflect back towards the laser.

\subsection{Mounting System}

As part of the design process, the system was designed to be mounted on different surfaces and devices. The first consideration was a fixed installation in a stationary location, such as hospital wards, work areas, or corridors, to perform targeted disinfection of a certain range of areas. Compared with UV LED lamps, lasers are more directional and take less time for disinfection, so the time for cleaning and disinfecting the room can be reduced. In a hospital, this can not only improve the efficiency of the hospital in the preparation of wards for new patients, but also avoids the potential harm to the human body from undifferentiated large-scale disinfection, such as that of UV LED lamps.

The whole system can also be implemented on a movable device to achieve disinfection in multiple areas, such as a cart or a drone. When mounting the system on a movable device, several problems need to be solved first. The first is the problem of power supply. In order to ensure long-term and large-scale disinfection, it is best for this equipment to be self-powered while moving. In addition, regarding the security issue, the whole system 
must be fully controllable. Finally, to address assembly problems, particular components must be designed to achieve a stable mounting of the entire optical system on the mobile device.

This mobile solution can be applied to several different solutions, such as cars or drones. In order to demonstrate the versatility of this design, we show it installed it on a hoverbike. This hoverbike can be ridden and flown at a low altitude with sufficient space to mount the entire system. We designed several components for mounting the whole system onto the hoverbike. In the design of mounting systems, the device was altered to include a different assembly that uses clips to mount the disinfection system onto the chassis of the hoverbike, thus adding the functionality of our UV disinfection system to the pre-existing device. The system was designed so that it can be used in a variety of settings to accomplish sanitation for a multitude of use cases; the hoverbike represents only one of these cases. This is a suitable method for demonstrating that the system can be implemented in several different ways, rather than just a stationary approach. Further details regarding our results on the hoverbike are shown in Section 3.3.

\section{Results and Analysis}

\subsection{Image Recognition Results}

The following results are from the SSD model discussed in the previous setup. The images used for testing were taken in a potential environment for deployment of the system.

Figure 2 shows the classification result of a chair based on the trained deep learning model. Figure $2 \mathrm{a}$ is the original image from the camera. The bounding box in Figure $2 \mathrm{~b}$ shows the result of recognition based on the neural network. The accuracy of the classification in the figure is $26 \%$. In order to show the label more clearly, we did not indicate the accuracy rate in the figure but discuss it here instead. It is worth noting that the definition of accuracy here is not the same as the classification accuracy of the entire network model. The accuracy discussed here is the probability that the detected object is in this class category. In contrast, the probability that the detected part in the bounding box in Figure $2 \mathrm{~b}$ is a door handle or a keyboard (other classes in Table 1 ) is far less than $26 \%$. The neural network will sort according to the probabilities of all the results and select the one with the highest probability as the predicted class category. Unless the most likely prediction result does not match the facts, for the entire neural network, this is still regarded as a correct prediction. The overall accuracy of the residual network was around $90 \%$, which shows the accuracy and feasibility of our experiments. Figure $2 c$ shows the UV disinfection of the surface of the detected objective based on the deep learning model.
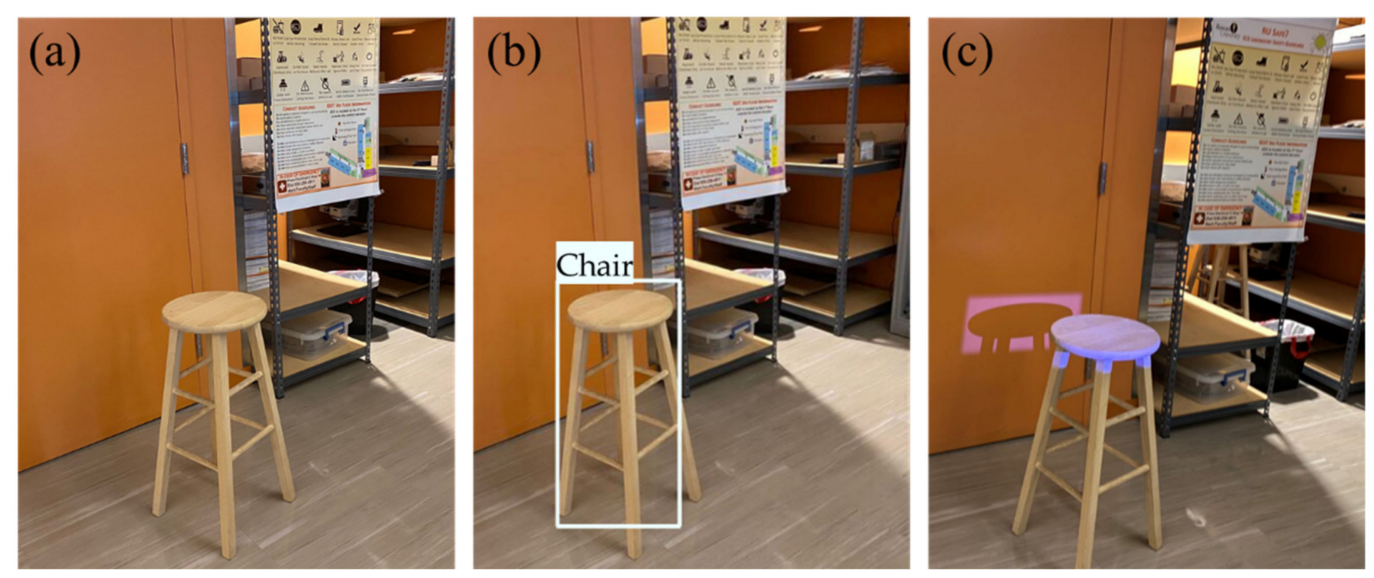

Figure 2. (a) Original image of a chair from a regular web/surveillance camera; (b) image recognition result with Deep Learning; (c) the area that needs to be disinfected is illuminated (violet area). 
Figure 3 shows the classification results of a person standing in front of a door. Figure $3 \mathrm{a}$ is the original image taken from camera. All the original images were resized to $640 \times 640$ resolution and fed into the network. Due to privacy protection, the face of the experimenter is blurred. Figure $3 \mathrm{~b}$ shows the predicted labels. Three classes were detected in the figure which are: Door handle $58 \%$, Person $23 \%$, and Table $14 \%$. It is worth noting that the network will output all the labels that are higher than the average probability $(12.5 \%)$. However, because the bounding box of Person in the middle overlaps with the bounding box of Table in the lower right corner, the system initiates the protection behavior and does not disinfect the lower right area. Only the area of door handle was disinfected. This shows that our system is protective against human contact.
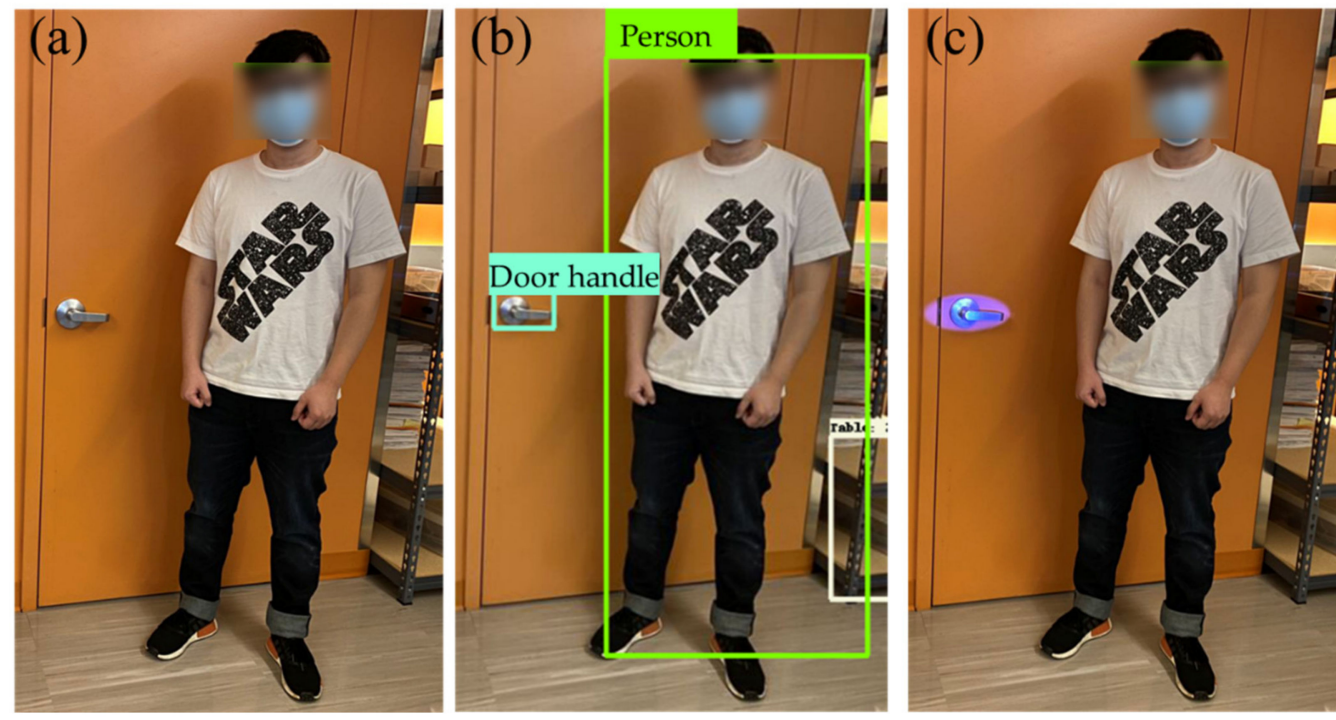

Figure 3. (a) Original image of a person standing in front of a door; (b) image recognition result with Deep Learning; (c) the area that needs to be disinfected is illuminated.

Figure 4 shows the classification results of a person sitting on a chair. Figure $4 \mathrm{a}$ is the original image taken from the camera. Due to privacy protection, the face of the experimenter is blurred. Figure $3 \mathrm{~b}$ shows the predicted labels. When the bounding box of Person overlaps with the bounding boxes of other categories in a large area, the predicted results completely cover the other classes in Table 1, except Person, thereby shutting down the entire system and protecting the human from the harm of the UV laser. The accuracy of the Person class in the figure is $32 \%$. This proves that our system has a very good protection against the special circumstances.
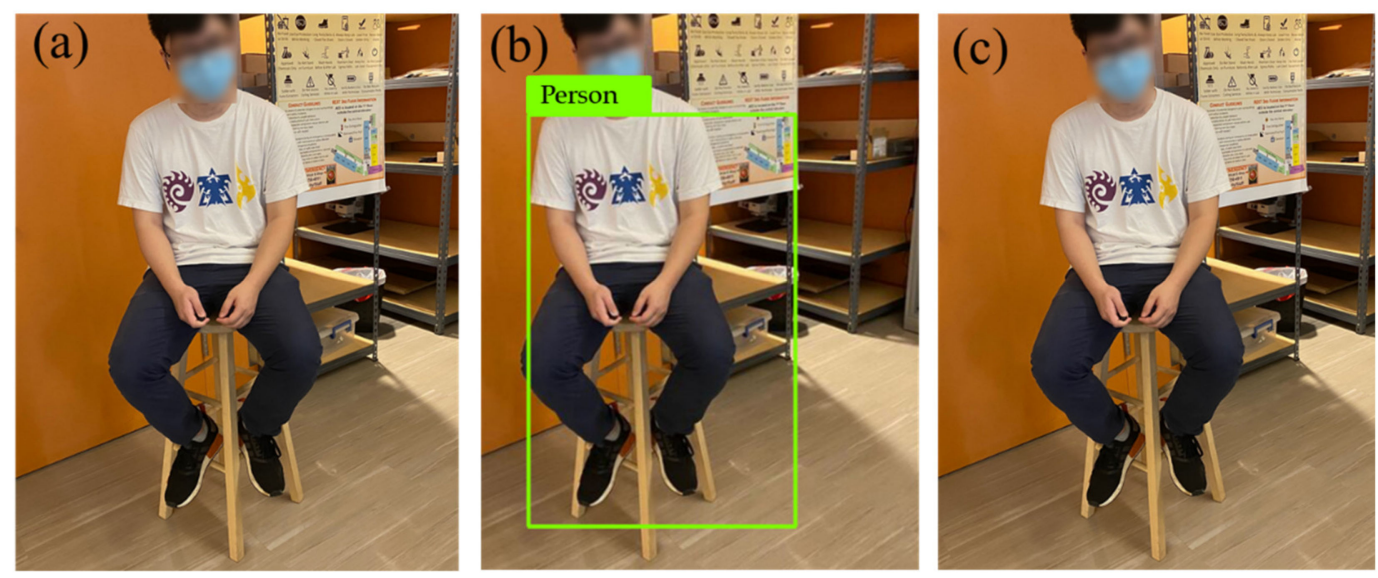

Figure 4. (a) Original image of a person sitting on a chair; (b) image recognition result with Deep Learning; (c) UV disinfection is turned off because a person has been detected. 


\subsection{Laser Galvanometer Results}

When the program determines the area that can be disinfected in the current area, the system starts the galvo mirror and laser, and disinfects the area. Due to the invisibility of the UV laser, to visualize the disinfection process, these tests were performed using a laser in the visible spectrum. These wavelengths are not effective for disinfection and were only used to show the effect.

Through the rapid vibrating mirror, the laser can scan a designated area to achieve the desired disinfection. The galvo system has a vibration frequency of up to $60 \mathrm{~Hz}$. Because the scanning method quickly moves the focus, the intensity of the laser in a short period is high enough for thorough disinfection. Figure 5 was created by overlaying the video frames of the system simulating disinfecting a section of the floor. Note: The gaps seen between the laser passes are due to the frame rate of the video; however, the laser beam did hit every point in the given area.

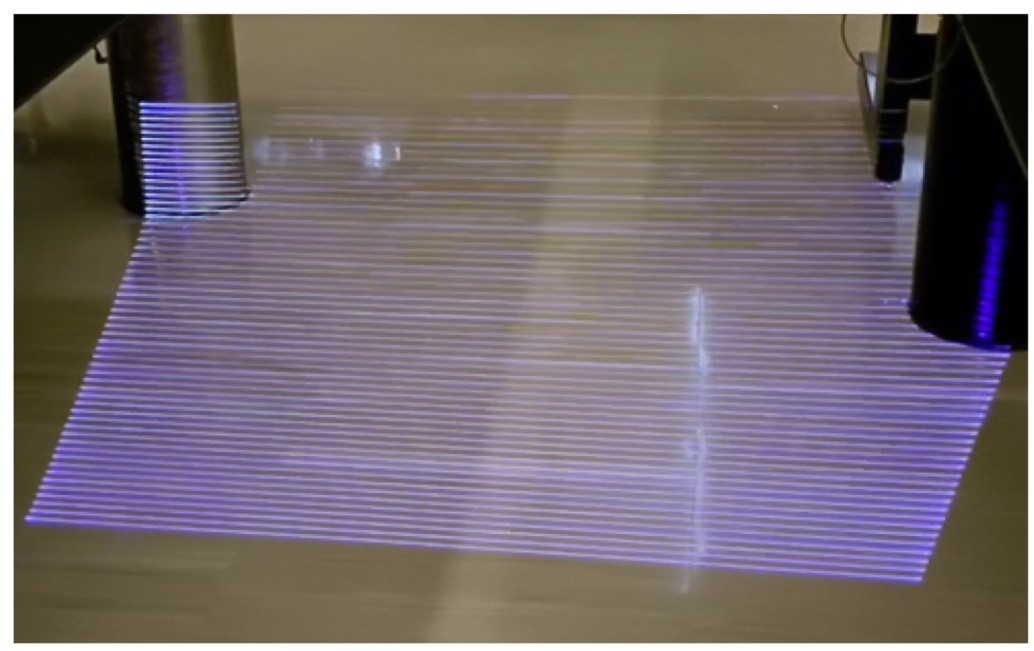

Figure 5. Laser galvanometer simulating sanitizing the ground.

\subsection{Simulated Use Case and Mounting System}

The system was designed so that it can be used in a variety of settings to accomplish sanitation for a multitude of use cases. The designed protype can perform a scan at an angle of $30^{\circ}$ at the rate of up to 100 cycles per second (Figure 6a). The existing system will be mounted on motorized rotation stages (Figure $6 \mathrm{~b}$ ), so the system can scan any directions in spherical coordinates. By adjusting the power of UV irradiation, the scanning distance of the system can range from a few millimeters to hundreds of meters.

Figure 6a shows a render of the device in its simplest form. It includes the laser, in addition to the focus and the enclosure for both devices. It also shows the galvanometer and a demonstration of how the laser can be angled. These components were commercially available [22] and configured to work with our design. Figure $6 \mathrm{~b}$ shows the device in Figure 6a, combined with a two-axis gimbal [23], to provide the widest possible range of motion. This model was made to show the capability of the system, even in a stationary location. 
(a)

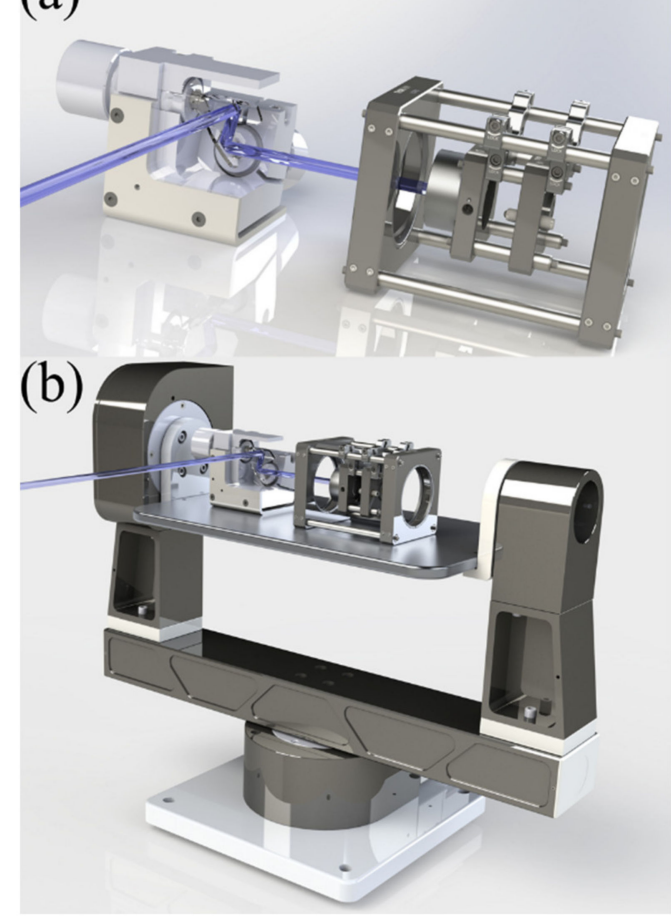

Figure 6. (a) Mounting system with galvo mirror and lens; (b) mounting system with added 2-axis gimbal. The transparent blue line represents the laser path.

For the mobile device mounting scenario, we also conducted numerous tests on a hoverbike to simulate all potential mobile devices, such as drones. The full hoverbike assembly is shown in the Figure 7 below.

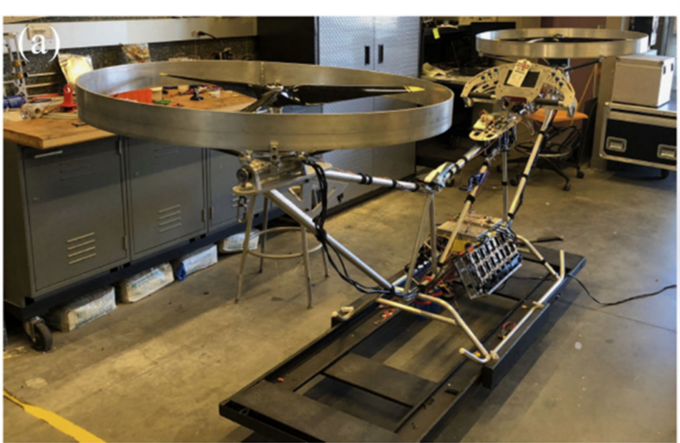

(b)

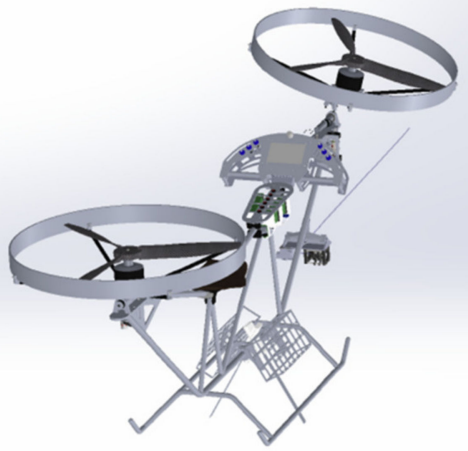

Figure 7. (a) Real shot of hoverbike; (b) 3D model of hoverbike.

The first priority when adding the laser system was ensuring that it was located so that it maintained the balance of the hoverbike while allowing the widest range of motion for the laser. Currently the laser is mounted to the front of the bike on the down tube. The details of the mounting are shown in the Figure 8 below: 

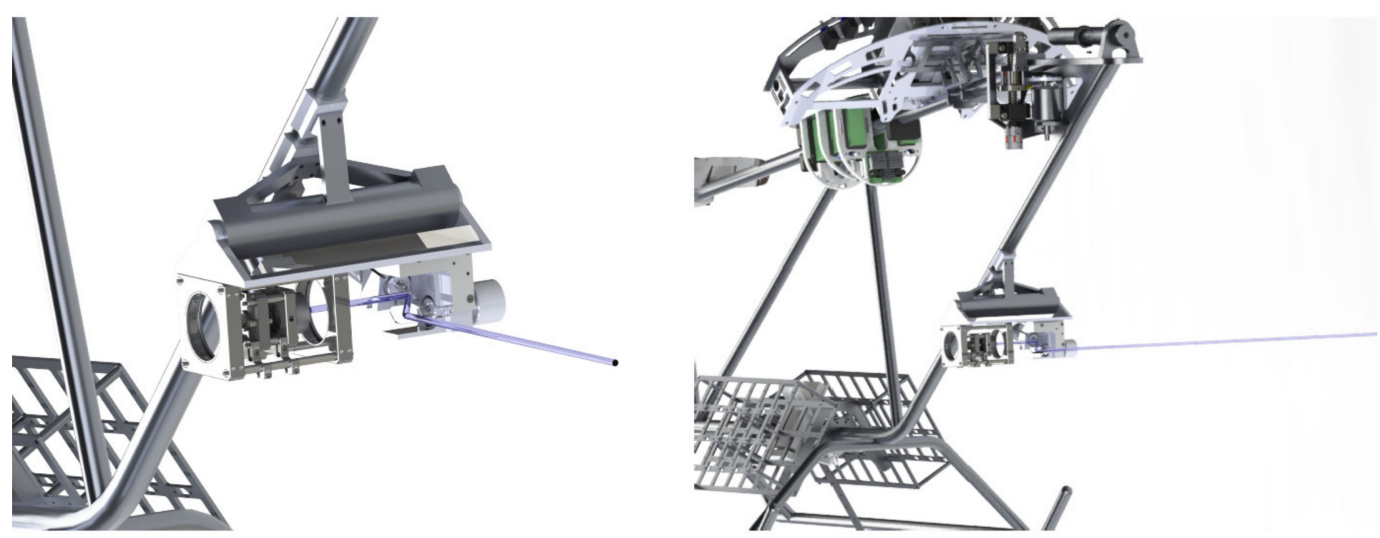

Figure 8. System mounted on the hoverbike. The transparent blue line represents the laser path.

This location was chosen because placing the system above the handlebars would interfere with the pilot's vision and the pilot would also block a wide portion of the laser's span. Under the hoverbike would also be optimal; however, this would not work with the current legs of the device and would be very vulnerable when landing. The selected location also gives the pilot the ability to aim the system in the desired location, so that the program can scan the desired location while avoiding unnecessary or unwanted surfaces such as pedestrians. More disinfection scenarios can be achieved by adding mounting systems adapted to different mobile devices such as a cart or a drone.

\section{Conclusions}

We proposed and demonstrated a deep learning controlled ultraviolet laser disinfection system based on a galvo mirror. This can achieve disinfection at multiple angles and in multiple scenarios via installation on different devices using the versatile mounting system. The use of a laser instead of a traditional UV LED allows for faster, more efficient, and safer disinfection because it is not indiscriminate in its use. The deep-learning network can achieve image recognition to identify the desired classes and humans. When these are in the same disinfection area, the process stops. However, when they are separate, the process can continue, thus ensuring that safety is paramount and that the entire system is able to function as intended.

Author Contributions: Conceptualization, T.S. and B.W.; methodology, B.W.; software, B.Z., T.D., S.F., N.P. and Z.S.; hover bike design, H.Z.; writing-original draft preparation, B.Z., T.D., S.F., N.P. and Z.S.; writing - review and editing, T.S. and B.W. All authors have read and agreed to the published version of the manuscript.

Funding: This research is funded by New Jersey Health Foundation, grant number PC 77-21.

Conflicts of Interest: The authors declare no conflict of interest.

\section{References}

1. Gidari, A.; Sabbatani, S.; Bastianelli, S.; Pierucci, S.; Busti, C.; Bartolini, D.; Stabile, A.M.; Monari, C.; Galli, F.; Rende, M.; et al. SARS-CoV-2 Survival on Surfaces and the Effect of UV-C Light. Viruses 2021, 13, 408. [CrossRef] [PubMed]

2. Mara, B.; Bianco, A.; Giovanni, P.; Adalberto, C.; Cavatorta, C.; Claudio, F.; Galli, P.; Luigi, L.; Manuela, L.; Enrico, T.; et al. UV-C irradiation is highly effective in inactivating SARS-CoV-2 replication. Sci. Rep. 2021, 11, 1-7.

3. Raeiszadeh, M.; Adeli, B. A critical review on ultraviolet disinfection systems against COVID-19 outbreak: Applicability, validation, and safety considerations. ACS Photonics 2020, 7, 2941-2951. [CrossRef]

4. Storm, N.; McKay, L.G.; Downs, S.N.; Johnson, R.I.; Birru, D.; de Samber, M.; Willaert, W.; Cennini, G.; Griffiths, A. Rapid and complete inactivation of SARS-CoV-2 by ultraviolet-C irradiation. Sci. Rep. 2020, 10, 1-5.

5. Inagaki, H.; Saito, A.; Sugiyama, H.; Okabayashi, T.; Fujimoto, S. Rapid inactivation of SARS-CoV-2 with deep-UV LED irradiation. Emerg. Microbes Infect. 2020, 9, 1744-1747. [CrossRef] [PubMed] 
6. Casini, B.; Tuvo, B.; Cristina, M.L.; Spagnolo, A.M.; Totaro, M.; Baggiani, A.; Privitera, G.P. Evaluation of an ultraviolet C (UV-C) light-emitting device for disinfection of high touch surfaces in hospital critical areas. Int. J. Environ. Res. Public Health 2019, 16, 3572. [CrossRef] [PubMed]

7. McDevitt, J.J.; Stephen, N.R.; Lewis, J.R. Aerosol susceptibility of influenza virus to UV-C light. Appl. Environ. Microbiol. 2012, 78, 1666-1669. [CrossRef] [PubMed]

8. Leung, K.C.P.; Ko, T.C.S. Improper use of the germicidal range ultraviolet lamp for household disinfection leading to phototoxicity in COVID-19 suspects. Cornea 2021, 40, 121-122. [CrossRef] [PubMed]

9. Boardman, E.A.; Huang, L.S.W.; Robson-Hemmings, J.J.; Smeeton, T.M.; Hooper, S.E.; Heffernan, J. Deep ultraviolet (UV-C) laser for sterilisation and fluorescence applications. Sharp Tech. Rep. 2012, 31. [CrossRef]

10. Sabino, C.P.; Sellera, F.P.; Sales-Medina, D.F.; Machado, R.R.G.; Durigon, E.L.; Freitas-Junior, L.H.; Ribeiro, M.S. UV-C (254 nm) lethal doses for SARS-CoV-2. Photodiagnosis Photodyn. Ther. 2020, 32, 101995. [CrossRef] [PubMed]

11. Tiseni, L.; Chiaradia, D.; Gabardi, M.; Solazzi, M.; Leonardis, D.; Frisoli, A. UV-C Mobile Robots with Optimized Path Planning: Algorithm Design and On-Field Measurements to Improve Surface Disinfection Against SARS-CoV-2. IEEE Robot. Autom. Mag. 2021, 28, 59-70. [CrossRef]

12. Chui, H.C.; Juang, S.S.Y.; Lin, H.Y.; Huang, C.H.; Liao, H.H. Ultraviolet Laser Sterilization System. U.S. Patent 9,061,083, 23 June 2015. Filed 7 July 2014.

13. Mackenzie, D. Ultraviolet light fights new virus. Engineering 2020, 6, 851. [CrossRef] [PubMed]

14. Zaccone, G.; Karim, M.R.; Menshawy, A. Deep Learning with TensorFlow; Packt Publishing Ltd.: Birmingham, UK, 2017.

15. Kuznetsova, A.; Rom, H.; Alldrin, N.; Uilings, J.; Krasin, I.; Pont-Tuset, J.; Kamali, S.; Popov, S.; Malloci, M.; Kolesnikov, A.; et al. The Open Images Dataset V4: Unified image classification, object detection, and visual relationship detection at scale. Int. J. Comput. Vis. 2020, 128, 1956-1981. [CrossRef]

16. He, K.; Zhang, X.; Ren, S.; Sun, J. Deep residual learning for image recognition. CVPR 2016, 770-778. [CrossRef]

17. Ren, S.; He, K.; Girshick, R.; Sun, J. Faster r-cnn: Towards real-time object detection with region proposal networks. NIPS 2015, 28, 91-99. [CrossRef] [PubMed]

18. Wen, L.; Li, X.; Gao, L. A transfer convolutional neural network for fault diagnosis based on ResNet-50. Neural Comput. Appl. 2020, 32, 6111-6124. [CrossRef]

19. Lin, T.Y.; Maire, M.; Belongie, S.; Hays, J.; Perona, P.; Ramanan, D.; Dollár, P.; Zitnick, C.L. Microsoft coco: Common objects in context. ECCV 2014, 740-755. [CrossRef]

20. He, T.; Zhang, Z.; Zhang, H.; Zhang, Z.; Xie, J.; Li, M. Bag of tricks for image classification with convolutional neural networks. CVPR 2019, 558-567.

21. He, K.; Zhang, X.; Ren, S.; Sun, J. Spatial pyramid pooling in deep convolutional networks for visual recognition. IEEE Trans. Pattern Anal. Mach. Intell. 2015, 37, 1904-1916. [CrossRef] [PubMed]

22. Thorlabs: Standard $60 \mathrm{~mm}$ Cage Plates. Available online: https://www.thorlabs.com/newgrouppage9.cfm?objectgroup_id=3021 (accessed on 4 March 2020).

23. Systems N: GM-12 Gimbal Mount. Available online: https://www.newmarksystems.com/gimbal-mounts/gm-12-gimbal/ (accessed on 2 February 2021). 